\title{
Iperplasia prostatica benigna sintomatica: ruolo degli inibitori della 5 -alfa-reduttasi nella prevenzione della ritenzione urinaria acuta e degli interventi chirurgici
}

\author{
Symptomatic benign prostatic hyperplasia: the role of 5-alpha-reductase \\ inhibitors in the prevention of acute urinary retention and surgical therapy
}

\section{Norma Marigliano ${ }^{a}$, Domenico Galasso ${ }^{\text {b,* }}$}

\author{
a Dipartimento di Farmacologia, Università degli Studi Magna Graecia, Catanzaro \\ b Direttore Emerito di Medicina Interna, Azienda Ospedaliera "Pugliese-Ciaccio", Catanzaro
}

Ricevuto il 22 novembre 2009; accettato il 22 luglio 2010

disponibile online il 19 novembre 2010

\section{KEYWORDS \\ Benign prostatic hyperplasia; 5-alpha-reductase inhibitors; Finasteride; Dutasteride. \\ * Corrispondenza: v.le Brutium 46/A - 88100 Catanzaro. \\ E-mail: domenico.galasso@libero.it (D. Galasso).}

\begin{abstract}
Summary Benign prostatic hyperplasia (BPH) is a disease that affects over $50 \%$ of males aged 50 years or older. In men aged $>80$ years, the incidence is $90 \%$. BPH occurs in $9-25 \%$ of males aged 40 to 79 years. Fifty percent of patients with BPH are symptomatic. The symptoms include reduced urinary flow, nocturia, defective bladder emptying, urinary hesitancy, and dysuria. Disease progression can be associated with acute urinary retention (AUR). Prostatic obstruction includes mechanical and dynamic components, the latter mediated by alpha-muscarinic receptors. Treatment with alpha-1-blockers (alfuzosin, doxazosin, tamsulosin, and terazosin) leads to rapid amelioration of symptoms and urinary flow, usually within one or two weeks. The 5-alpha reductase inhibitors (5-ARIs) are "disease-modifying drugs." They control the growth of the prostate by blocking the conversion of testosterone into dihydrotestosterone (DHT). Finasteride is a $5-A R I$ that is selective for type 2 receptors. Dutasteride is a powerful inhibitor of both 5- alpha reductase isoforms (type 1 and 2) and produces more complete suppression of DHT synthesis than finasteride. Dutasteride also has a much longer half-life than finasteride (five weeks versus five to six hours). The authors review the results of clinical trials involving finasteride and dutasteride, with and without alpha-1-blockers, highlighting the important role of dutasteride in improving acute urinary retention and eliminating the need for surgical therapy. (c) 2010 Elsevier Srl. All rights reserved.
\end{abstract}




\section{Introduzione}

L'iperplasia prostatica benigna è una malattia che colpisce oltre il $50 \%$ degli uomini di età $\geq 50$ anni e quasi il $90 \%$ degli ottantenni [1]. È presente nel 9-25\% della popolazione maschile tra i 40-79 anni. Il 50\% dei pazienti è sintomatico. Il $17 \%$ è in età lavorativa e riferisce che i sintomi pregiudicano il rendimento sul lavoro [2]. Infatti, si riducono del $25 \%$ l'attività lavorativa, del $16 \%$ la validità e l'energia, del $14 \%$ il ruolo emozionale, del $14 \%$ il benessere generale, dell' $11 \%$ le forze fisiche, dell' $8 \%$ le funzioni sociali, del $7 \%$ il benessere mentale [3]. L'iperplasia prostatica benigna comporta 4,5 milioni di visite ambulatoriali e una spesa annua di 1,1 miliardi di dollari per servizi medici diretti, oltre ai costi relativi alla terapia farmacologica dei pazienti non ospedalizzati [4]; tali spese complessive sono destinate a crescere, considerato l'andamento demografico dei soggetti di età $\geq 64$ anni in una proiezione a 10 anni.

Polimorfa è la sintomatologia specifica: dalla riduzione del flusso minzionale alla sensazione di svuotamento incompleto, al ritardo nell'inizio della minzione, alla nicturia, alla disuria. Col progredire della malattia, si può verificare ritenzione urinaria acuta. L'ostruzione prostatica, oltre a una componente meccanica, riconosce una componente dinamica, controllata dai recettori alfa ${ }_{1}$ muscarinici.

\section{Studi clinici}

Gli alfa ${ }_{1}$-litici (doxazosina, terazosina, tamsulosina, alfuzosina) comportano un rapido miglioramento dei sintomi, e del flusso, in 1-2 settimane. Confrontando terazosina e tamsulosina, il miglioramento del symptom score è sovrapponibile; la differenza è nella rapidità d'azione: tamsulosina raggiunge il risultato prima [5]. L'efficacia degli alfa ${ }_{1}$-litici si esplica nella riduzione dell'American Urological Association-Symptom Score (AUA-SS), con incremento del flusso urinario massimo [6] L'efficacia si limita al rilasciamento della muscolatura liscia, senza alcun effetto sulla riduzione del volume prostatico [7]. Tra gli effetti collaterali sono da ricordare vertigini, cefalea, palpitazioni, lipotimia, sincope per la terazosina e la doxazosina; eiaculazione retrograda, ridotto volume dell'eiaculato e possibile aneiaculazione per la tamsulosina $[8,9]$.

Gli inibitori della 5-alfa-reduttasi (5-ARI) sono farmaci "modificatori di malattia"; convertono il testosterone in diidrotestosterone (DHT), intervenendo così nel controllo della crescita della prostata [10]. La finasteride è un 5-ARI selettivo per i recettori di tipo 2 . La dutasteride è un potente inibitore di entrambe le isoforme della 5-alfa-reduttasi (tipo 1 e 2) e determina una maggiore e più consistente soppressione del DHT, rispetto alla finasteride; inoltre ha un'emivita molto più lunga (5 settimane vs 5-6 ore) [11].

La loro efficacia si è dimostrata nel ridurre il volume prostatico del $20-25 \%$, con miglioramento dei sintomi, minore rischio di ritenzione urinaria acuta e minore ricorso alla chirurgia [12]. I benefici clinici compaiono dopo 6-12 mesi dall'inizio della terapia.

Lo studio VA-COOP, della durata di 52 settimane e condotto su 1.229 pazienti, ha valutato finasteride $5 \mathrm{mg}$, terazosina $10 \mathrm{mg}$ e una combinazione ottenendo una variazione media del punteggio dei sintomi di $-3,2$ con finasteride (non significativo), -6 con terazosina ( $p<0,001),-6,1$ con la combinazione ( $p<0,001)$. Le variazioni del volume prostatico, calcolate in base alle medie alla settimana 52 e alla settimana 24 , sono state di 0,5 per terazosina,- 6,1 per

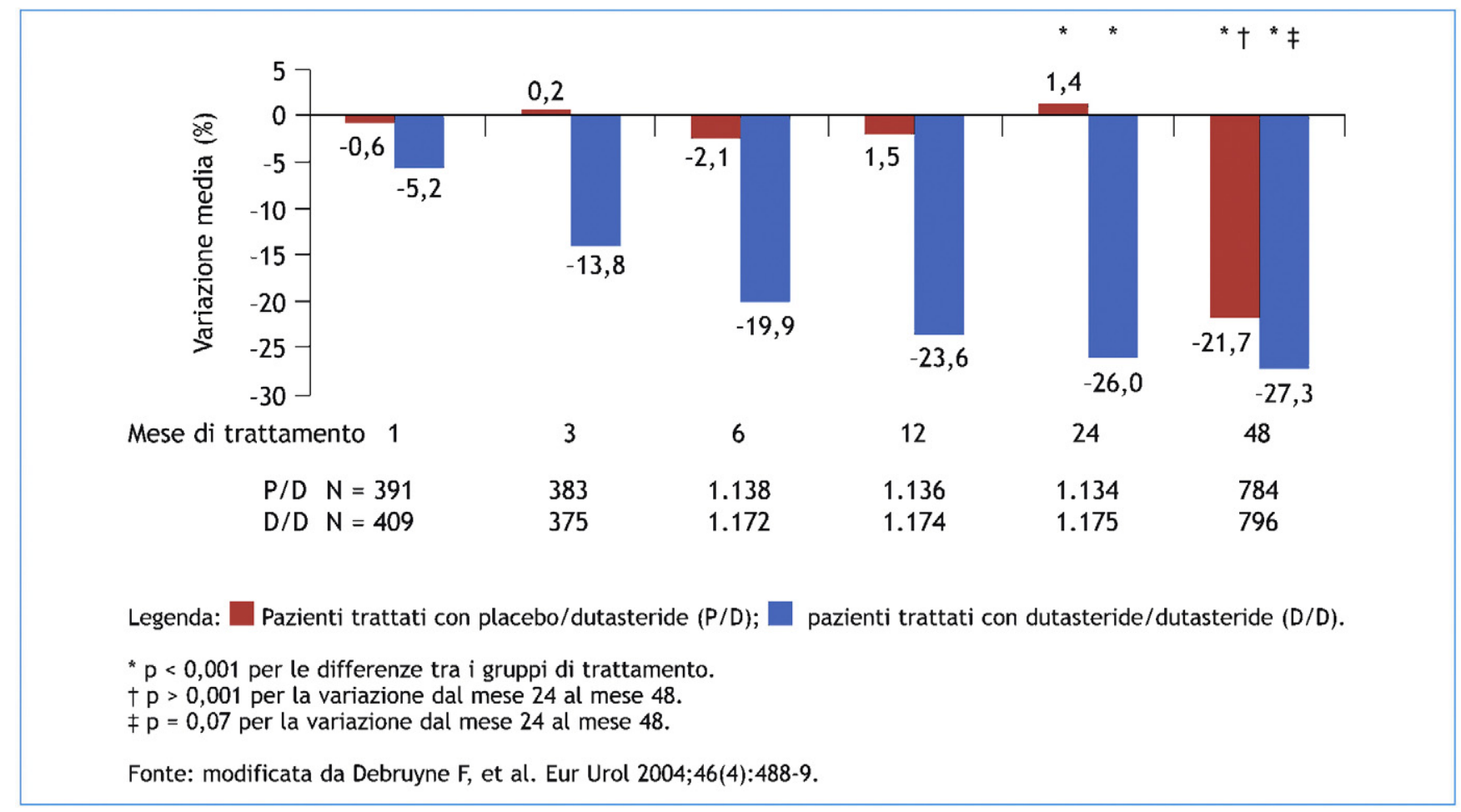

Figura 1 Variazione media (\%) del volume prostatico dall'inizio dello studio fino a 48 mesi. 
finasteride $(p<0,001)$ e $-7,0$ per la combinazione $(p<0,001)$ [13].

Di grande rilevanza sono tre studi clinici di fase III, ARIA3001, ARIA3002 e ARIB3003, della durata di 2 anni, randomizzati, controllati verso placebo, condotti su larga scala, prolungati poi di altri 2 anni, in aperto, per verificare l'efficacia e la sicurezza a lungo termine di dutasteride. Sono stati verificati significativi miglioramenti del punteggio sintomatologico, secondo l'American Urological AssociationSymptom Index (AUA-SI), e del flusso urinario massimo $\left(\mathrm{Q}_{\max }\right)$, rispettivamente tra il $24^{\circ}$ e il $48^{\circ}$ mese, in entrambi i gruppi di studio. In particolare, al $48^{\circ}$ mese, i pazienti del gruppo dutasteride/dutasteride hanno mostrato miglioramenti significativamente maggiori rispetto ai soggetti del gruppo placebo/dutasteride; inoltre, solo un'esigua percentuale di pazienti, durante la fase in aperto, ha presentato ritenzione urinaria acuta ed è stata sottoposta a interventi chirurgici correlati all'iperplasia prostatica benigna [14] (figg. 1-4).

Lo studio MTOPS (Medical Therapy of Prostatic Symptoms) è stato condotto per 4,5 anni e ha arruolato 3.047 pazienti. Sono stati utilizzate finasteride, doxazosina e la

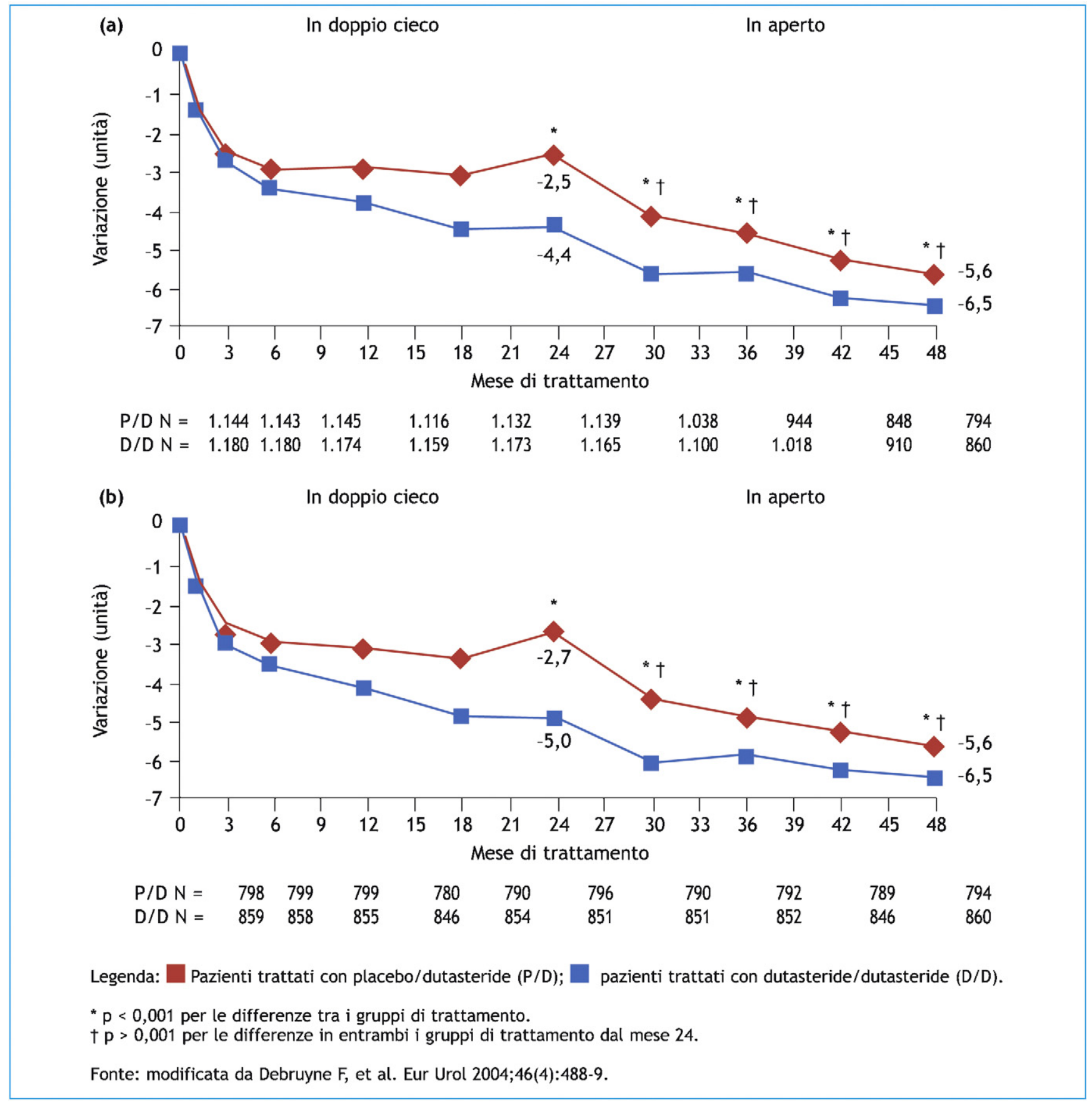

Figura 2 Variazione media del punteggio all'American Urological Association-Symptom Index dall'inizio dello studio fino a 48 mesi: (a) popolazione ITT in aperto; (b) popolazione che ha completato il trattamento. 


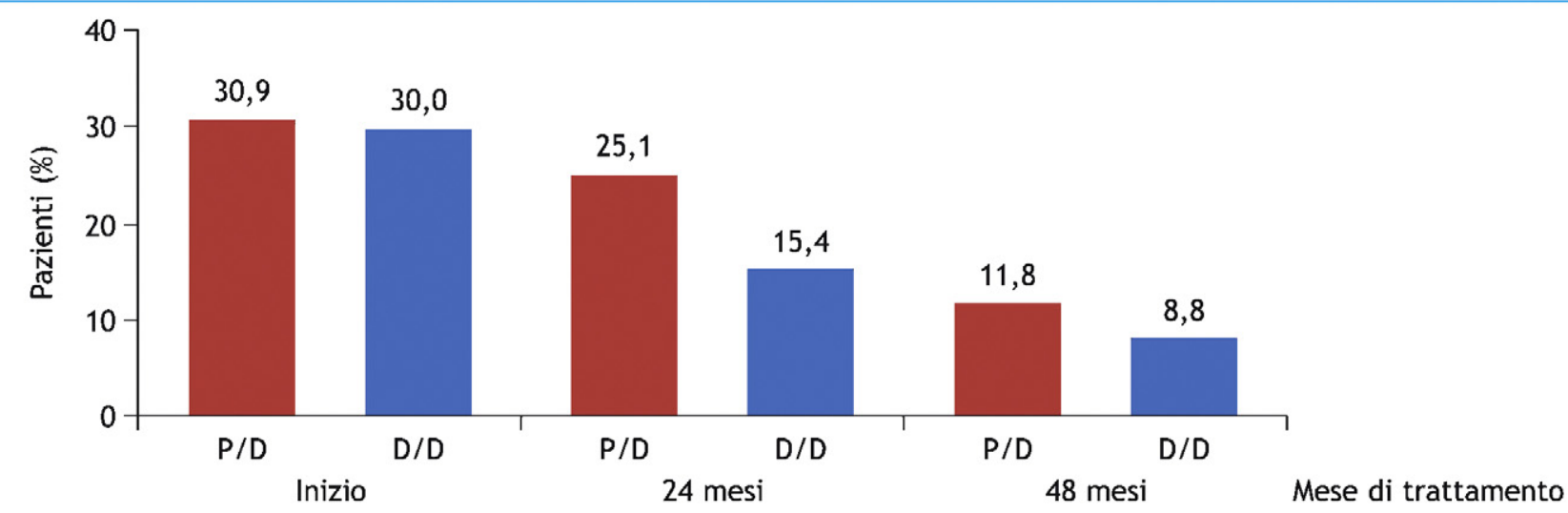

Fonte: modificata da Debruyne F, et al. Eur Urol 2004;46(4):488-9.

Figura 3 Percentuali di soggetti del gruppo placebo/dutasteride (P/D) e del gruppo dutasteride/dutasteride (D/D) con sintomi urinari gravi (punteggio all'American Urological Association-Symptom Index compreso tra 20 e 35 calcolato all'inizio dello studio, a 24 mesi e a 48 mesi).

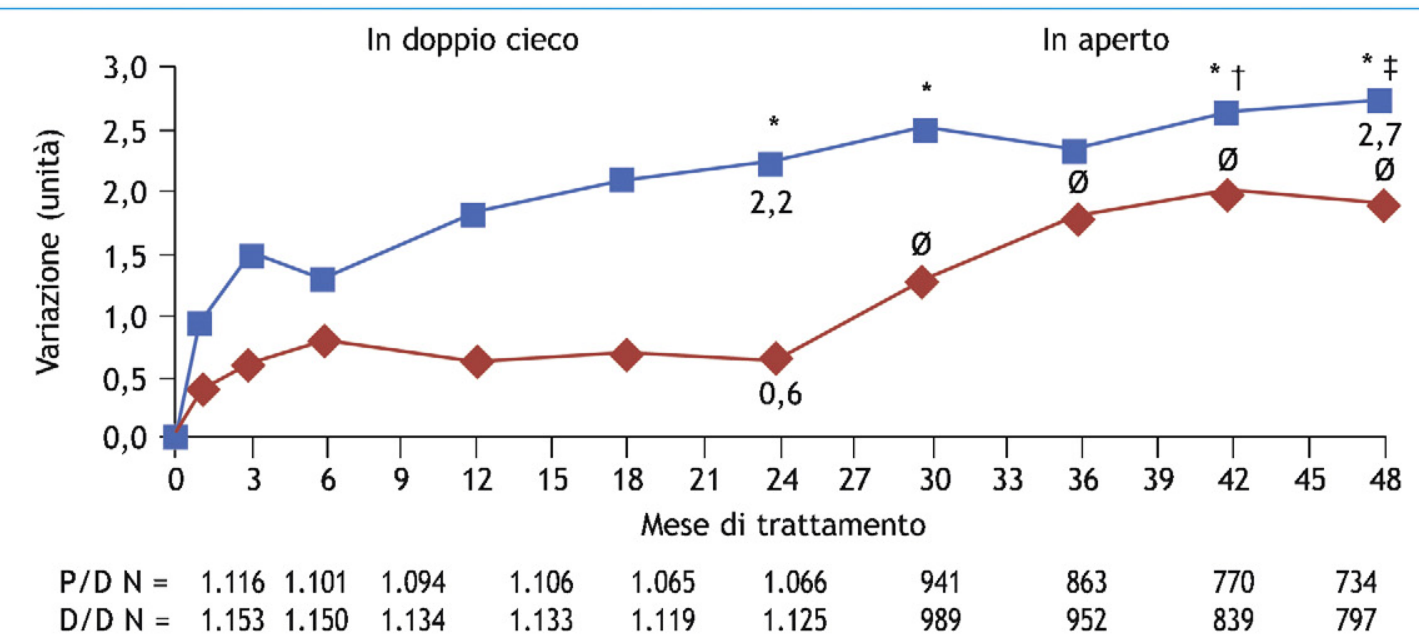

Legenda: $\square$ Pazienti trattati con placebo/dutasteride (P/D); $\square$ pazienti trattati con dutasteride/dutasteride (D/D).

${ }^{*} p<0,001$ per le differenze tra i gruppi di trattamento.

$\dagger p=0,042$ per le differenze tra $i$ gruppi di trattamento.

$\neq \mathrm{p}>0,032$ per le differenze tra i gruppi di trattamento.

Fonte: modificata da Debruyne F, et al. Eur Urol 2004;46(4):488-9.

Figura 4 Variazione media del flusso urinario massimo dall'inizio dello studio fino a 48 mesi.

combinazione. Sono stati valutati dopo 1 anno e dopo 4 anni: il punteggio allo AUA-SS, che può variare da 0 in assenza di sintomi a 35 in presenza di sintomi gravi; il flusso urinario massimale; i valori di antigene prostatico specifico (PSA) sierico (variazione percentuale rispetto al basale).

- Per lo AUA-SS, la doxazosina ha registrato una variazione mediana all'anno $1 \mathrm{di}-6,0$ ( $p<0,001$ ) e all'anno $4 \mathrm{di}-6,0$ $(p<0,001)$. Per la finasteride all'anno $1, p$ era uguale a 0,777 e all'anno 4 a 0,035. Per la combinazione si otteneva $-6,0$ all'anno 1 ( $p<0,001)$ e $-7,0$ ( $<<0,001)$ all'anno 4.
- Il flusso urinario massimo (misurato in $\mathrm{mL} / \mathrm{sec}$ ) si incrementava $(\mathrm{p}<0,001)$ sia a 1 anno sia a 4 anni con la doxazosina e con la combinazione; tuttavia il suo aumento non si verificava con la sola finasteride.

- Per il terzo parametro, la variazione del PSA, si rilevava una riduzione significativa $(\mathrm{p}<0,001)$ sia a 1 anno sia a 4 anni con finasteride e con la combinazione, ma non con sola doxazosina [15].

Lo studio CombAT, a lungo termine, ha verificato il ruolo della terapia di combinazione dutasteride $(0,5 \mathrm{mg})$ e 
l'alfa-litico tamsulosina $(0,4 \mathrm{mg})$ in pazienti di età $\geq 50$ anni con diagnosi di iperplasia prostatica benigna, sintomi da moderati a gravi, punteggio all'International Prostate Symptom Score (IPSS) $\geq 12$, ingrossamento prostatico $\geq 30 \mathrm{cc}$, PSA $\geq 1,5 \mathrm{ng} / \mathrm{mL}$ [16], picco di flusso massimo $\geq 5 \mathrm{e} \leq 15 \mathrm{~mL} / \mathrm{sec}$ e volume minimo residuo $\geq 125 \mathrm{~mL}$. Gli obiettivi di efficacia erano valutati a 2 anni. L'endpoint primario era costituito dalla variazione del punteggio all'IPSS rispetto al basale [17].

La variazione dell'IPSS dal basale, tra il valore al tempo $0 \mathrm{e}$ quello a 24 mesi, ha identificato una significativa variazione ( $<<0,001$ ) per la combinazione vs dutasteride da sola, o vs tamsulosina, dal mese 9 al mese 24. Sono state osservate: una variazione del picco di flusso massimo dal basale con un valore di $p=0,006$ per la combinazione vs dutasteride e vs tamsulosina; una significativa variazione dello stato di salute della prostata ( $p<0,001$ ) per la combinazione vs dutasteride e vs tamsulosina; una significativa variazione del volume prostatico ( $p<0,001)$ per la combinazione vs tamsulosina; una significativa variazione percentuale media del volume ( $p<0,001$ ) per la combinazione vs tamsulosina. La dutasteride ha determinato una diminuzione della concentrazione sierica di PSA del 50\% dopo 6 mesi in pazienti con iperplasia prostatica benigna, anche in presenza di tumore della prostata. I valori di PSA dovranno essere raddoppiati per poterli paragonare con i valori normali di soggetti non sottoposti al trattamento, pur riconoscendo la sensibilità e specificità del test del PSA nel rilevare il tumore prostatico.

In un altro lavoro [19], i pazienti trattati con dutasteride hanno riportato un punteggio migliore allo AUA-SI rispetto ai pazienti che avevano ricevuto finasteride $(6,1$ vs 3,3$)$ e un miglioramento clinico più rapido [18]. Valutando poi le percentuali di ritenzione urinaria acuta, e gli interventi chirurgici alla prostata, l'incidenza per i pazienti in trattamento con dutasteride era più bassa: $5,3 \%$ vs $8,3 \%, p<0,0207$ [19]. Tra gli effetti collaterali, la disfunzione erettile, la riduzione della libido, la ginecomastia.

\section{Discussione}

Il trattamento con la dutasteride, inibitore di entrambe le isoforme della 5-alfa-reduttasi, nei vari studi induce una prolungata e consistente riduzione della concentrazione sierica di $\mathrm{DHT}$, assieme a una riduzione continuativa del volume prostatico totale, con un miglioramento costante della sintomatologia e del flusso urinario. La diminuzione dei livelli di DHT si verifica nel $90 \%$ dei pazienti, con un decremento $\geq 90 \%$ e una probabilità di raggiungere la riduzione massima del volume prostatico totale. Inoltre, migliora costantemente il punteggio sintomatologico allo AUA-SI, con attenuazione clinicamente rilevante della sintomatologia e riduzione significativamente più consistente del volume. Buona la tollerabilità, con una riduzione nel corso del tempo dell'incidenza dei più comuni eventi avversi a carico della sfera sessuale. Fa eccezione la ginecomastia, la cui incidenza si mantiene bassa ma costante nel tempo, tra l'1,3\% dopo 1 anno e l'1,8\% dopo 3 anni.

\section{Conclusioni}

Il trattamento a lungo termine con dutasteride, nei soggetti con iperplasia prostatica benigna sintomatica, dà luogo a un miglioramento continuativo dei sintomi e del flusso urinario, con un'ulteriore riduzione del volume prostatico totale. La riduzione del rischio di ritenzione urinaria acuta, e di intervento chirurgico correlato alla patologia, si è osservata costantemente nel tempo; ciò contestualmente alla buona tollerabilità nel trattamento a lungo termine. I vantaggi sul decorso della malattia sono da considerarsi rilevanti, per il singolo paziente, e significativi sul versante delle spese mediche generali (ospedalizzazioni, visite ambulatoriali, utilizzo di risorse).

\section{Conflitto di interesse}

Gli autori dichiarano di essere esenti da conflitto di interessi.

\section{Bibliografia}

[1] Naslund MJ, Issa MM, Grogg AL, Eaddy MT, Black L. Clinical and economic outcomes in patients treated for enlarged prostate. Am J Manag Care 2006;12(4 Suppl):S111-6.

[2] Garraway WM, Russell EB, Lee RJ, et al. Impact of previously unrecognized benign prostatic hyperplasia on the daily activities of middle-aged and elderly men. $\mathrm{Br} \mathrm{J}$ Gen Pract 1993;43(373):318-21.

[3] Welch G, Weinger K, Barry MJ. Quality-of-life impact of lower urinary tract symptom severity: results from the Health Professionals Follow-up Study. Urology 2002;59(2):245-50.

[4] Wei JT, Calhoun E, Jacobsen SJ. Urologic diseases in America project: benign prostatic hyperplasia. J Urol 2005;173(4):125661.

[5] Lowe F, Narayan P, Djavan B. Prospective, randomised, multicentre trial to evaluate the rapidity of onset and side effect profile of tamsulosin (TAM) vs terazosin (TER) in men with Benign Prostatic Hyperplasia (BPH). Eur Urol Suppl 2002;1(1): 108.

[6] Narayan P, Evans CP, Moon T. Long-term safety and efficacy of tamsulosin for the treatment of lower urinary tract symptoms associated with benign prostatic hyperplasia. J Urol 2003;170 (2 Pt 1):498-502.

[7] Thomas K, Chow K, Kirby RS. Acute urinary retention: a review of the aetiology and management. Prostate Cancer Prostatic Dis 2004;7(1):32-7.

[8] Lepor $\mathrm{H}$. The pathophysiology of lower urinary tract symptoms in the ageing male population. $\mathrm{Br} \mathrm{J}$ Urol 1998;81(Suppl 1): 29-33.

[9] van Kerrebroeck P, Jardin A, Laval KU, van Cangh P. Efficacy and safety of a new prolonged release formulation of alfuzosin $10 \mathrm{mg}$ once daily versus alfuzosin $2.5 \mathrm{mg}$ thrice daily and placebo in patients with symptomatic benign prostatic hyperplasia. ALFORTI Study Group. Eur Urol 2000;37(3):306-13.

[10] Fenter TC, Davis EA, Shah MB, Lin PJ. Dutasteride vs finasteride: assessment of differences in acute urinary retention rates and surgical risk outcomes in an elderly population aged $>$ or $=65$ years. Am J Manag Care 2008;14(5 Suppl 2):S154-9.

[11] Clark RV, Hermann DJ, Cunningham GR, Wilson TH, Morrill BB, Hobbs $S$. Marked suppression of dihydrotestosterone in men with benign prostatic hyperplasia by dutasteride, a dual 5alpha-reductase inhibitor. J Clin Endocrinol Metab 2004;89(5): 2179-84.

[12] Roehrborn CG, Boyle P, Nickel JC, Hoefner K, Andriole G, ARIA3001 ARIA3002 and ARIA3003 Study Investigators. Efficacy and safety of a dual inhibitor of 5-alpha-reductase types 1 and 2 (dutasteride) in men with benign prostatic hyperplasia. Urology 2002;60(3):434-41. 
[13] Lepor H, Williford WO, Barry MJ, Haakenson C, Jones K. The impact of medical therapy on bother due to symptoms, quality of life and global outcome, and factors predicting response. Veterans Affairs Cooperative Studies Benign Prostatic Hyperplasia Study Group. J Urol 1998;160(4):1358-67.

[14] Debruyne F, Barkin J, van Erps P, Reis M, Tammela TL, Roehrborn C, ARIA3001, ARIA3002 and ARIB3003 Study Investigators. Efficacy and safety of long-term treatment with the dual 5 alphareductase inhibitor dutasteride in men with symptomatic benign prostatic hyperplasia. Eur Urol 2004;46(4):488-9.

[15] McConnell JD, Roehrborn CG, Bautista OM, et al., Medical Therapy of Prostatic Symptoms (MTOPS) Research Group. The long-term effect of doxazosin, finasteride, and combination therapy on the clinical progression of benign prostatic hyperplasia. N Engl J Med 2003;349(25):2387-98.

[16] Siami P, Roehrborn CG, Barkin J, et al., CombAT Study Group. Combination therapy with dutasteride and tamsulosin in men with moderate-to-severe benign prostatic hyperplasia and prostate enlargement: the CombAT (Combination of Avodart and Tamsulosin) trial rationale and study design. Contemp Clin Trials 2007;28(6):770-9.

[17] Roehrborn CG, Siami P, Barkin J, et al., CombAT Study Group. The effects of dutasteride, tamsulosin and combination therapy on lower urinary tract symptoms in men with benign prostatic hyperplasia and prostatic enlargement: 2 -year results from the CombAT study. J Urol 2008;179(2):616-21.

[18] Hagerty JA, Ginsberg PC, Harkaway RC. A prospective comparative study of the onset of syntomatic benefit of dutasteride versus finasteride in men with benign prostatic hyperplasia in clinical practice. Annual Meeting of the American Urology Association. May 8, 2004; San Francisco.

[19] Issa MM, Runken MC, Grogg AL, Shah MB. A large retrospective analysis of acute urinary retention and prostate-related surgery in BPH patients treated with 5-alpha reductase inhibitors: dutasteride versus finasteride. Am J Manag Care 2007;13(Suppl 1): S10-6. 